\title{
Investigation on papillomavirus infection in dromedary camels in Al-Ahsa, Saudi Arabia
}

\author{
Abdelmalik Ibrahim Khalafalla ${ }^{1,2, *}$, Ramadan Omer Ramadan ${ }^{3}$, Annabel Rector ${ }^{4}$ and Seif Barakat ${ }^{5}$ \\ ${ }^{1}$ Camel Research Centre, King Faisal University, Al Ahsa, Saudi Arabia \\ ${ }^{2}$ Department of Microbiology, Faculty of Veterinary Medicine, University of Khartoum, Sudan \\ ${ }^{3}$ Department of Clinical Studies, College of Veterinary Medicine, King Faisal University, Al Ahsa, Saudi Arabia \\ ${ }^{4}$ KU Leuven, Department of Microbiology and Immunology, Laboratory of Clinical \& Epidemiological Virology, \\ B-3000 Leuven, Belgium \\ ${ }^{5}$ Department of Pathology, College of Veterinary Medicine, King Faisal University, Saudi Arabia
}

\begin{abstract}
We investigated two outbreaks of papillomatosis between 2013 and 2015 in Al Ahsa region of eastern Saudi Arabia involving fourteen dromedary camels. The disease affected both young and adult animals and occurred in coincidence with demodectic mange infestation. Diagnosis was made based on gross and histopathological characteristics of the wart lesion and was confirmed by PCR. Rolling circle amplification followed by degenerate primer PCR and sequencing of the amplicons revealed the presence of both Camelus dromedarius papillomavirus types 1 and 2, previously identified in infected dromedaries in Sudan.
\end{abstract}

Keywords: Al Ahsa, Dromedary camels, Infection, Papillomavirus, Saudi Arabia.

\section{Introduction}

Papillomas (warts) are benign epithelial proliferations of the skin and mucous membranes caused by papillomaviruses (PVs) and are seen worldwide in man and a number of animals. PVs constitute a diverse group of small $(52-55 \mathrm{~nm})$, non-enveloped, circular double-stranded DNA viruses, classified in the Papillomaviridae family. These viruses are known as species-specific and, in experimental conditions, do not infect another host than their natural one (Campo, 2006).

The first report of papillomatosis associated with PV in dromedaries was published in 1990 (Munz et al., 1990) and showed that dromedary camels in central Somalia aged 6 months to 2 years were primarily affected. Later, cases of papillomatosis in young dromedary camels have also been reported from Kenya (Dioli and Stimmelmayr, 1992), United Arab Emirates (Wernery and Kaaden, 1995) and Sudan (Khalafalla et al., 1998; Ure et al., 2011).

PVs were found associated with a $2 \mathrm{~kg}$ wart-like growth on the right fetlock joint of a dromedary camel in India (Sadana et al., 1980) and a corneal papilloma mass in the left eye of a 15-year old dromedary male with a history of chronic severe keratoconjuctivitis (Kilic et al., 2010). Papillomatosis has also been shown in South American camelids in llamas and alpacas (Schulman et al., 2003). The majority of the described camel papillomatosis cases is usually seen in young animals and occurs in the late rainy season, coinciding with outbreaks of camel contagious ecthyma and camel pox (Munz et al., 1990; Khalafalla et al., 1998).

Currently, the genomes of two Camelus dromedaries PV types (type 1, CdPV1, and type 2, CdPV2) have been fully characterized and genetically grouped within the genus Deltapapillomavirus (Ure et al., 2011). The two genotypes were isolated from a cauliflower-like nodule and a round, oval raised nodule, respectively, observed in 3 and 7 months old dromedary camels in Sudan.

Here, we describe the clinic-pathological features of cases of papillomatosis, as well as the genetic identification of the associated CdPVs from Saudi Arabia.

\section{Ethical statement}

\section{Material and Methods}

Skin specimens were collected according to the Guidelines for Ethical Conduct for Use and Care of Animals in Research of King Faisal University (KFU), Saudi Arabia and approved by the KFU Ethics Care Committee.

Case description of camel papillomatosis in Al-Ahsa Group I:

In November 2013, two young dromedary camels (aged 14 months) of the Maghateer breed (Table 1) were referred to the Veterinary teaching hospital of KFU for hernia surgery in one of them.

\section{Group II:}

In October 2014, an outbreak of skin warts occurred in a camel farm of KFU in Al-Ahsa province of eastern 
KSA. This farm includes 68 dromedary camels of different breeds; two were at one month of age, ten were less than two years of age, 18 were at 2-4 years of age and the rest were adults (more than four years of age). The farm is located about $2 \mathrm{~km}$ away from the Veterinary hospital and perform zero grazing with no access to the pasture or contact with other camel herds. History of the disease, including age and breed of affected animals as well as the type and number of wart lesions is shown in Table 1.

\section{Specimen collection}

Specimens of six warts, one from each affected camel, were surgically removed and divided into two parts. One part was processed for DNA extraction and the other was fixed in $10 \%$ buffered formalin for histopathology.

\section{Histopathology}

For histopathological examination the specimens were trimmed and put in vacuum infiltrating tissue processing machine (Tissue- Tik VIP 5Jr. Japan) and then embedded in paraffin wax by SLEE MPS/C machine, Germany. Specimens were then waxed in blocks and sectioned at $5 \mu \mathrm{m}$ by LEICA RM 2235 microtome, Germany. Specimens were then mounted on glass slides and stained with Haematoxylin and Eosin (H\&E) for histopathological examination (Kiernan, 1999).

\section{DNA extraction}

Wart tissues were cut into 2-3 mm pieces by a pair of sterile scissors and a $20 \%$ suspension of the minced tissues was made in tris-EDTA (TE) buffer ( $\mathrm{pH}$ 7.4). Specimens were then homogenized using a mechanical homogenizer (TissueRuptor, Qiagen, Germany) and centrifuged at $1500 \mathrm{~g}$ for $10 \mathrm{~min}$ at $4^{\circ} \mathrm{C}$. Total viral DNA was extracted from $200 \mu 1$ of each specimen's supernatant using GF-1 Viral Nucleic Acid Extraction Kit (Vivantis Technologies, Malaysia) according to manufacturer instructions.

\section{PCR}

As the differential diagnosis for camel papillomatosis includes camelpox and contagious ecthyma, all 6 DNA samples were screened using a multiplex gel-based PCR (Khalafalla et al., 2015), which uses the FAP59/64 consensus primers originally designed from L1 gene conserved region of PV (Forslund et al., 1999). Additionally, DNA samples were screened using PV specific degenerate primer pair AR-E1F2/AR-E1R3 (371 bp) in accordance with Rector et al. (2004a).

\section{Rolling circle amplification}

Multiply primed rolling circle amplification (RCA) was performed with an Illustra TempliPhi amplification kit (GE Healthcare Life Sciences) following a protocol that was optimized for the amplification of papillomaviral complete genomic DNA (Rector et al., 2004b; Stevens et al., 2010). One microliter of sample (extracted DNA or water as a negative control) was added to $5 \mu 1$ of TempliPhi sample buffer, containing exonuclease protected random hexamers. The sample was than denatured at $95^{\circ} \mathrm{C}$ for 3 minutes and afterwards placed on ice. A premix consisting of $5 \mu \mathrm{l}$ of TempliPhi reaction buffer, $0.2 \mu 1$ of TempliPhi enzyme mix containing $\varphi 29$ DNA polymerase, and $0.2 \mu \mathrm{l}$ of extra deoxynucleotides (dNTPs, each $25 \mathrm{mM}$ ) was prepared and added to each sample $(5 \mu \mathrm{l})$. The reaction was incubated overnight (approximately 16 hours) at $30^{\circ} \mathrm{C}$. Reactions were then put on ice, and subsequently heated to $65^{\circ} \mathrm{C}$ for 10 minutes to inactivate the $\varphi 29$ DNA polymerase.

To investigate whether papillomaviral DNA was amplified, $2 \mu 1$ of the RCA product was digested with a restriction enzyme panel consisting of BamHI, EcoRI, SalI, HindIII and HincII. The digestion products were run on a $0.8 \%$ agarose gel to check for the presence of a DNA band consistent with full-length PV DNA $(\sim 8 \mathrm{~kb})$, or multiple bands with sizes adding up to this length.

Table 1. History of dromedary Papillomatosis in two herds in Al Ahsa, eastern Saudi Arabia.

\begin{tabular}{ccccll}
\hline $\begin{array}{c}\text { Animal } \\
\text { group }\end{array}$ & $\begin{array}{c}\text { Animal } \\
\text { No. }\end{array}$ & Age & Breed & Type of wart lesion (number) & $\begin{array}{l}\text { Total number of wart lesions; site } \\
\text { (number per site) }\end{array}$ \\
\hline I & Rm1 & 14 months & Maghateer & Cauliflower-like (3) & 3: upper lip (1), lower lip (2) \\
I & Rm2 & 14 months & Maghateer & Cauliflower-like (2) & 2: upper lip (2) \\
II & CRC1 & 6 years & Majaheem & Cauliflower-like (8); nodular (12) & 20: upper lip (10), lower lip (6), eyelid (4) \\
II & CRC2 & 5 years & Majaheem & Cauliflower-like (2); nodular (4) & 6: upper lip (3), lower lip (3) \\
II & CRC3 & 3 years & Majaheem & Cauliflower-like (1) & 1: upper lip \\
II & CRC4 & 6 years & Majaheem & Cauliflower-like (4); nodular (3) & 7: nostril (3), upper lip (2), lower lip (2) \\
II & CRC5 & 7 years & Majaheem & Nodular (19) & 19: upper lip (12), lower lip (7) \\
II & CRC6 & 4 years & Majaheem & Cauliflower-like (2) & 2: upper lip (1), mandible (1) \\
II & CRC7 & 14 months & Majaheem & Cauliflower-like (3); nodular (2) & 5: upper lip (2), mandible (2), nostril (1) \\
II & CRC8 & 2 years & Maghateer & Nodular (2) & 2: lower lip \\
II & CRC22 & 6 years & Maghateer & Cauliflower-like (1); nodular (7) & 3: upper lip (7), mandible (1) \\
II & CRC28 & 22 months & Maghateer & Cauliflower-like (1) & 1: upper lip \\
II & CRC42 & 13 months & Maghateer & Nodular (40) & 40: upper lip \\
II & CRC77 & 13 months & Majaheem & Nodular (28) & 28: upper (22) and lower lips (6) \\
\hline
\end{tabular}




\section{Degenerate primer PCR and sequencing}

Degenerate papillomavirus-specific primers were used to screen RCA products. The following primer pairs were used:FAP59/FAP64 (Forslund et al., 1999), ARL1F1/AR-L1R3 (Rector et al., 2004a), and ARE1F2/AR-E1R3 (Rector et al., 2004a).

Previously unpublished primer pairs AR-L1F11/ARL1R10 and AR-E1F14/AR-E1R12, developed in conserved L1 and E1 regions of ungulate PVs, were also tested (Table 2).

Amplification was performed with the Qiagen OneStep RT-PCR kit following manufacturer's instructions, using a concentration of $2.4 \mu \mathrm{M}$ of forward and reverse primer, and $1 \mu \mathrm{l}$ of RCA product as sample in a final reaction volume of $25 \mu \mathrm{l}$.

PCR products were checked on agarose gel and further sequenced bi-directionally on an ABI Prism 3100 Genetic Analyzer (Applied Biosystems, Life Technologies). Sequences were analyzed by similarity searches using NCBI BLAST.

Table 2. Degenerate primers of amplification of Papilloma viral E1 and L1 sequences.

\begin{tabular}{ll}
\hline Primer & \multicolumn{1}{c}{ Sequence (5'-3') } \\
\hline FAP59 & TAACWGTNGGNCAYCCWTATT \\
FAP64 & CCWATATCWVHCATNTCNCCATC \\
AR-L1F1 & TTDCAGATGGCNGTNTGGCT \\
AR-L1R3 & CATRTCHCCATCYTCWAT \\
AR-L1F11 & GGDGAYATGATGGAHATWGG \\
AR-L1R10 & CCATTRTTCATDCCCTGDGC \\
AR-E1F2 & ATGGTNCAGTGGGCNTATGA \\
AR-E1R3 & TTNCCWSTATTNGGNGGNCC \\
AR-E1F14 & CTTTGACACAYAYCTCAGAAAY \\
AR-E1R12 & AGVTCTAANCGYYCCCATARCCTT \\
\hline
\end{tabular}

\section{Results}

\section{Gross morphology of lesions}

The two animals in group I, belonging to a dromedary camel farm in Riyadh region of central Saudi Arabia, showed numerous nodular and cauliflower-like proliferations on the lips and nostrils, which had first been noticed 4 weeks prior to sampling. In group 2, the disease occurred in epizootics form involving 14 (Table 1) out of 81 dromedary camels, giving a morbidity rate of $17.3 \%$. The number of warts per animal varied from one to 20 , with a size ranging from 0.3 to $2.2 \mathrm{~cm}$. They were particularly located on the lips, eyelids, nostrils and mandible (Fig. 1). The lesions first appeared as rosy hyperemic elevations of the skin and developed into solid cauliflower-like exophytic growth taking the skin or a darker colour in the majority of the animals. The cauliflower-like papillary masses were dark in color with approximate size of $2 \mathrm{~cm}$ in diameter (Fig. 2).

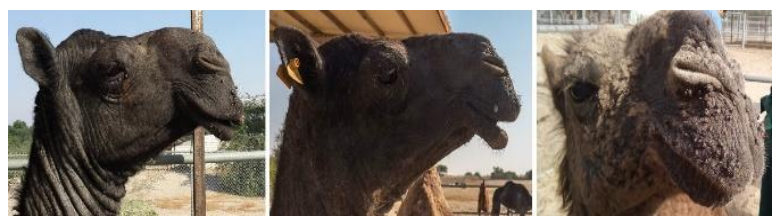

Fig. 1. Papillomatosis in dromedary camels in Al Ahsa, Saudi Arabia. Cauliflower-like and multiple nodular Papillomas on the face that vary in number and size.
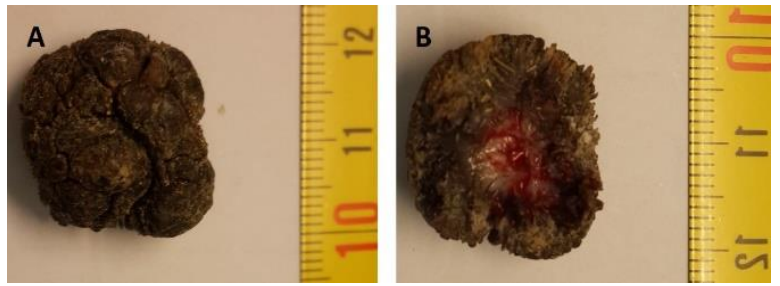

Fig. 2. Gross appearance of the papilloma wart showing cauliflower protruded dark in color. The diameter was $2 \mathrm{~cm}$.

Some animals however showed rounded raised nodules instead. Data on the age of camels, breed, clinical type and numbers of warts are summarized in Table 1 . The disease, which began in October 3 continued through October and ended in early November 2014. Later in March 2016, two young dromedaries aged 13 months and from the non-affected pens in the same farm developed typical papilloma lesions.

\section{Histopathological findings}

Sections of all the biopsy samples collected from camels with wart-like lesions showed identical histopathological features characterized by multiple papillary proliferation, hyperkeratosis and acanthosis (Fig. 3 and 4).

Diagnostics by PCR and Rolling circle amplification Pan PV PCR amplification was positive in eight animals, including Rm1, Rm2, CRC3, 5, 7, 8, 22, 42 and CRC 77 (Table 3).

Possible PV genomic amplification by RCA was observed for samples Rm1 and Rm2. These RCA products were submitted to degenerate primer PCR, and PV specific sequences could be amplified for all these samples (Table 3).

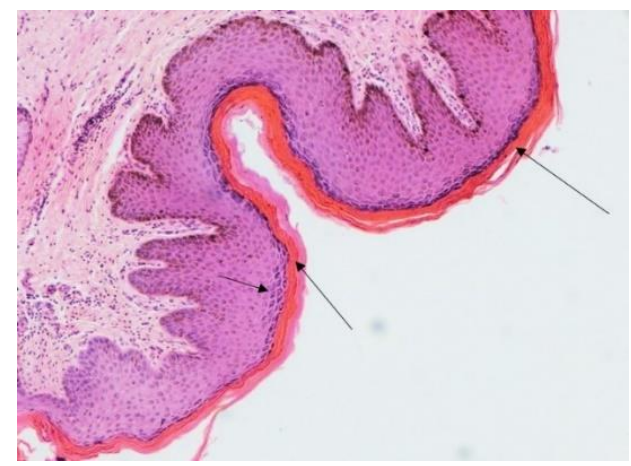

Fig. 3. Skin section of camel, showing hyperkeratosis (long arrows), hydropic degeneration of keratinocytes, acanthosis (short arrow), and mild infiltration of inflammatory cells. 
Table 3. Sequence data generated from Papilloma lesions collected from dromedary camels in Saudi Arabia.

\begin{tabular}{ccclc}
\hline Sample & Sequence & \# nt* & Most similar to papillomavirus type & $\%$ nt identity \\
\hline \multirow{3}{*}{ Rm1 } & ARL1F1/ARL1R3 & 530 & Camelus dromedarius papillomavirus 1 & $97 \%$ \\
& ARL1F11/ARL1R10 & 328 & Camelus dromedarius papillomavirus 1 & $97 \%$ \\
& ARE1F14/ARE1R12 & 205 & Camelus dromedarius papillomavirus 1 & $99 \%$ \\
Rm2 & ARL1F1/ARL1R3 & 537 & Camelus dromedarius papillomavirus 1 & $98 \%$ \\
CRC 77 & ARL1F11/ARL1R10 & 289 & Camelus dromedarius papillomavirus 1 & $98 \%$ \\
CRC 3 & FAP59/FAP64 & 434 & Camelus dromedarius papillomavirus 2 & $98 \%$ \\
\hline
\end{tabular}

*length of the amplicon.

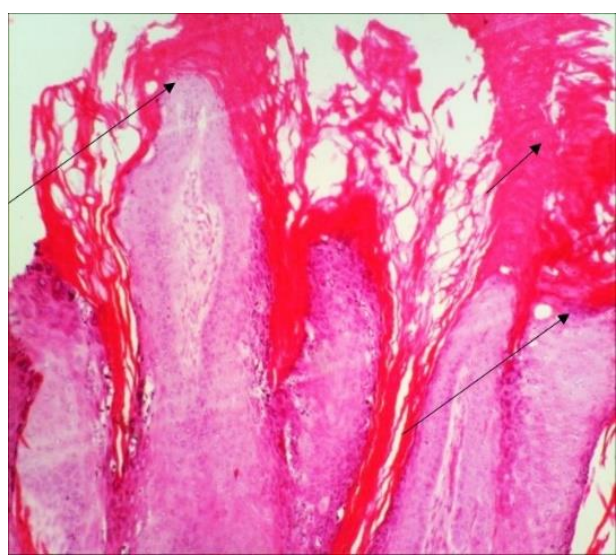

Fig. 4. Skin section of camel (Epidermis), showing multiple papillary proliferations (long arrows), and the epidermis is irregularly thickened and covered by a thick layer of hyperkeratosis (short arrow).

\section{Discussion}

We investigated PV infection in two groups of dromedary camels in Al-Ahsa province of eastern Saudi Arabia during 2013 and 2015. The diagnosis was done based on clinical findings, histopathology and PCR testing. While we were limited to gather enough information with group I, we could collect more data from group II.

Of interest, the disease affected predominantly camels severely infested with mange. Further parasitological investigations (data not shown) revealed that the mange mites were of the Demodex species. The preferred site of the burrowing mite of the genus Demodex is at the hair follicles and sebaceous glands of the skin. Camel mange is an extremely contagious skin disease, characterized by scab formation, pruritic dermatitis, thickening and corrugation of skin and hair loss, and caused by the parasitic mite (Mochabo et al., 2005).

Camels in the five other pens did not develop papillomatosis. We therefore suggest that mange was the major predisposing factor for the PV infection in dromedary camels. However, it is also possible that some animals in this herd had other underlying defects predisposing them to more severe infections with several pathogens, such as mange mites and PV. In a previous epidemiological study, we observed that most cases of PV infections in the Sudan were associated with contagious ecthyma virus infection (Khalafalla et al., 1998). Dioli and Stimmelmayr (1992) in Kenya also observed association between papillomatosis and camelpox virus (CMLV) infection. In line with this, coinfection of PV, ortho- and parapoxviruses in cattle has been described in Italy (Scagliarini et al., 2016).

Our observations raise many questions in the understanding of risk factors of PV infection in camels. It is well documented that transmission of PVs is usually horizontal and occurs via contact, abrasions or micro-lesions of the skin and mucosa, but vertical and iatrogenic transmissions have also been reported, as well as mechanical by arthropods (Campo and Bastianello, 2004).

Breaks in the integrity of the epidermis are likely to facilitate entry of the virus to the basal layers of the skin, hereby allowing infection of the epithelial stem cell which results in permanent viral replication in the epidermis.

Another finding of interest is the development of cutaneous papillomatosis in both adult and young camels while in most previous reports the affected groups were young dromedary camels [3-7 months (Ure et al., 2011), 3-14 months (Khalafalla et al., 1998) and 18-24 months (Oryan et al., 2011)]. All PV sequences showed high degrees of similarity to previously identified PV types. A new PV type is defined as a cloned full-length papillomaviral genome, whose L1 nucleotide sequence is at least $10 \%$ dissimilar from that of any other PV type (de Villiers et al., 2004; Bernard et al., 2010). Although no complete L1 ORFs were sequenced in this study, the partial L1 sequences retrieved from the camel samples showed at least $96 \%$ identity to Camelus dromedarius papillomavirus types 1 and 2, indicating the presence of subtypes or variants thereof, rather than new papillomavirus types.

Partial papillomaviral L1 sequences were obtained from 4 samples in the present study. Three animals were infected with Camelus dromedarius papillomavirus type 1 and one was infected with Camelus dromedarius papillomavirus type 2. As these two genotypes were previously detected in dromedary camels of Sudan, our results points to a wide 
distribution of these genotypes that also involve camels of the Arabian Peninsula. In contrast, the study of Ure et al. (2011) revealed the presence of two different PV species in only two samples taken from an affected herd, leading the authors to speculate that a wide diversity of PVs exists in dromedaries (Ure et al., 2011). It is noteworthy that in our present study only the same two previously identified Camelus dromedaries papillomavirus types were found. This might point towards a prevalence of these types, but does not exclude the possibility that dromedary camels can be infected by a wide diversity of papillomavirus types, as it has been demonstrated in most animal species that were intensively studied. In cattle, for instance, 15 bovine papillomaviruses (BPVs) have been completely genetically characterized (Papillomavirus Genome database, https://pave.niaid.nih.gov) but only BPV1 and BPV2 are broadly present while the other types are only found occasionally (Grindatto et al., 2015). In order to unravel the complete genomic diversity of camel dromedary PVs, further investigation of a large number of samples, preferably taken from different herds, is necessary.

Of interest, Camelus dromedarius papillomavirus type 2 was detected in a young camel, which showed the disease 13 months after the initial outbreak in farm II. While we only detected genotype 1 in the initial outbreak of this herd, it remains difficult to determine whether this genotype 2 was already circulating at that time but remained undetected or whether it represents a novel infection.

\section{Conclusions}

We investigated cases of warts or papillomatosis in camels in Saudi Arabia. Clinical findings, gross and histopathological features suggested papillomavirus infection while PCR and partial genome sequencing confirmed the diagnosis. Both genotypes 1 and 2, previously detected in Sudan, were responsible for these outbreaks.

\section{Acknowlegemets}

We thank Dr. Marzook Al-Eknah, Director, Camel Research Center, King Faisal University for support during sample collection and Dr Sophie Duraffour, Bernhard-Nocht Institute for Tropical Medicine, Hamburg, Germany for reading the manuscript.

\section{Conflict of interest}

The authors declare that there is no conflict of interests.

\section{References}

Bernard, H.U., Burk, R.D., Chen, Z., van Doorslaer, K., zur Hausen, H. and de Villiers, E.M. 2010. Classification of papillomaviruses (PVs) based on 189 PV types and proposal of taxonomic amendments. Virology 401, 70-79.

Campo, M.S. 2006. Bovine papillomavirus: old system, new lessons? In: Papillomavirus Research from
Natural History to Vaccines and Beyond. Campo MS, editor. Caister Academic Press, Norwich, UK, pp: 373-383.

Campo, S. and Bastianello, S.S. 2004. Papillomavirus infections In: J.A.W. Coetzer \& R.C Tustin (eds.), Infectious Diseases of Livestock, with Special Reference to Southern Africa (second ed.), Oxford University Press Southern Africa, Cape Town, South Africa.

de Villiers, E.M., Fauquet, C., Broker, T.R., Bernard, H.U. and zur Hausen, H. 2004. Classification of papillomaviruses. Virology 324, 17-27.

Dioli, M. and Stimmelmayr, R. 1992. Important camel diseases. In: The One Humped Camel in Eastern Africa, A. Pictorial Guide to Diseases, Health Care and Management. H. J. Schwarts and M. Dioli, editors. Verlag Josef Margrat Scientific Books, pp: 155-164.

Forslund, O., Antonsson, A., Nordin, P., Stenquist, B. and Hansson, B.G. 1999. A broad range of human papillomavirus types detected with a general PCR method suitable for analysis of cutaneous tumours and normal skin. J. Gen. Virol. 80, 2437-2443.

Grindatto, A., Ferraro, G., Varello, K., Crescio, M.I., Miceli, I., Bozzetta, E., Goria, M. and Nappi, R. 2015. Molecular and histological characterization of bovine papillomavirus in North West Italy. Vet. Microbiol. 180, 113-117.

Khalafalla, A.I., Abbas, Z. and Mohamed, M.E.H. 1998. Camel papillomatosis in the Sudan. J. Camel Pract. Res. 5, 157-159.

Khalafalla, A.I., Al-Busada, K.A. and El-Sabagh, I.M. 2015. Multiplex PCR for rapid diagnosis and differentiation of pox and pox-like diseases in dromedary Camels. Virol. J. 7(12), 102-109.

Kiernan, J.A. 1999. Histological and histochemical methods: Theory and practice. $3^{\text {rd }}$ ed. Butterworth Heinemann, Oxford, UK, pp: 502.

Kilic, N., Toplu, N., Aydog, A., Yaygıngu, R. and Ozsoy SY. 2010. Corneal papilloma associated with papillomavirus in a one-humped camel (Camelus dromedarius). Vet. Ophthalmol. 13(Suppl. 1), 100-102.

Mochabo, K.O., Kitala, P.M., Gathura, P.B., Ogara, W.O., Catley, A., Eregae, E.M. and Kaitho, T.D. 2005. Community perception of important camel diseases in Lapur division of Turkana district, Kenya. Trop. Anim. Health Prod. 37, 187-204.

Munz, E., Moallin, A. S., Mahnel, H. and Reimann, M., 1990. Camel papillomatosis in Somalia. Zentralbl Veterinarmed B 37, 191-196.

Oryan, A., Hashemnia, M., Mohammadalipour, A. and Gowharinia, M. 2011. Gross and histopathological characteristics of fibropapilloma in camels (Camelus dromedarius). J. Camel Pract. Res. 18, 65-68. 
Rector, A., Bossart, G.D., Ghim, S.J., Sundberg, J.P., Jenson, A.B. and Van Ranst, M. 2004a. Characterization of a novel close-to-root papillomavirus from a Florida manatee by using multiply primed rolling-circle amplification: Trichechus manatus latirostris papillomavirus type 1. J. Virol. 78, 12698-12702.

Rector, A., Tachezy, R. and Van Ranst, M. 2004b. A sequence-independent strategy for detection and cloning of circular DNA virus genomes by using multiply primed rolling-circle amplification. J. Virol. 78, 4993-4998.

Sadana, J.R., Mahajan, S.K. and Satija, K.C. 1980. Note on papilloma in a camel. Indian J. Anim. Sci. 50, 793-794.

Scagliarini, A., Casà, G., Trentin, B., Gallina, L., Savini, F., Morent, M., Lavazza, A., Puleio, R., Buttaci, C., Cannella, V., Purpari, G., Di Marco, P., Piquemal, D. and Guercio, A. 2016. Evidence of zoonotic Poxviridae coinfections in clinically diagnosed papillomas using a newly developed mini-array test. J. Vet. Diagn. Invest. 1, 59-64.

Schulman, F.Y., Krafft, A.E., Janczewski, T., Reupert, R., Jackson, K. and Garner, M.M. 2003. Camelid mucocutaneous fibropapillomas: clinicopathologic findings and association with papillomavirus. Vet. Pathol. 140, 103-107.

Stevens, H., Rector, A. and Van Ranst, M. 2010. Multiply primed rolling-circle amplification method for the amplification of circular DNA viruses. Cold Spring Harbor protocols 2010, pdb prot5415.

Ure, A.E., Elfadl, A.K., Khalafalla, A.I., Gameel, A.A.R., Dillner, J. and Forslund, O. 2011. Characterization of complete genomes of Camelus dromedarius papillomavirus 1 and 2. J. Gen. Virol. 8, 1769-1777.

Wernery, U. and Kaaden, O.R. 1995. Infectious Diseases of Camelids. Blackwell WissenschaftsVerlag, Berlin. 